\section{Production of Chemotactic Activity for Polymorphonuclear Leukocytes by Cultured Rat Hepatocytes Exposed to Ethanol}

H. Daniel Perez, F. Joseph Roll, D. Montgomery Bissell, Steven Shak, and Ira M. Goldstein Rosalind Russell Arthritis Research Laboratory and Liver Center, Department of Medicine, University of California, Medical Service, San Francisco General Hospital, San Francisco, California 94110
A polymorphonuclear leukocytes. As a possible explanation for this phenomenon, we have found that ethanol stimulates cultured rat hepatocytes to generate potent chemotactic activity. Hepatocytes (>99\% pure), isolated from the livers of Sprague-Dawley rats, responded to incubation with ethanol $(2.0-10 \mathrm{mM})$ by releasing chemotactic activity for human polymorphonuclear leukocytes into culture supernatants in a time- and concentration-dependent fashion. Chemotactic activity was maximal after incubation of hepatocytes with $10 \mathrm{mM}$ ethanol for $6 \mathrm{~h}$. It was undetectable in the absence of ethanol and was reduced in the presence of either the alcohol dehydrogenase inhibitor, 4-methylpyrazole, or the acetaldehyde dehydrogenase inhibitor, cyanamide. Ethanol failed to stimulate generation of chemotactic activity by either rat dermal fibroblasts, hepatic sinusoidal endothelial cells, or Kupffer cells. The chemotactic activity generated by ethanol-treated rat hepatocytes was recovered from culture supernatants in the lipid phase after extraction with chloroform/methanol. Thin-layer

Portions of this work were presented at the Annual Meeting of the Association of American Physicians, Washington, DC, 1983, and published in preliminary form (Trans. Assoc. Am. Phys. 96:56-64, 1983).

This work was done during the tenure, by Dr. Perez, of an Established Investigatorship from the American Heart Association, with funds contributed in part by the American Heart Association of San Francisco. Address all correspondence to Dr. Perez.

Received for publication 27 February 1984 and in revised form 7 May 1984

J. Clin. Invest.

(C) The American Society for Clinical Investigation, Inc

0021-9738/84/10/1350/08 \$1.00

Volume 74, October 1984, 1350-1357 chromatography and high performance liquid chromatography of chloroform/methanol extracts demonstrated that the chemotactic factor probably is a polar lipid. This chemotactic lipid may account, in part, for the leukocytic infiltration of the liver parenchyma that is observed during the course of acute alcoholic hepatitis.

\section{Introduction}

Ethanol provokes acute inflammation and tissue injury in the liver. The term "alcoholic hepatitis" denotes a disease characterized clinically by moderate to severe impairment of liver function secondary to excessive and continuous intake of ethanol. The pathology of ethanol-related liver disease includes, among other changes (e.g., fatty metamorphosis, hepatocellular swelling and necrosis, formation of Mallory bodies), infiltration of the centrilobular zone of the liver with polymorphonuclear leukocytes (PMN) ${ }^{1}$ and mononuclear cells (1). If biopsy specimens are obtained early in the course of the disease, PMN appear to be the predominant cell type (2) and often are seen surrounding Mallory bodies that have been extruded from dead liver cells (1). Some investigators have attributed the presence of PMN infiltrates to the ability of Mallory (hyaline) bodies to generate chemotactic factors. Leevy et al. (3) reported that although purified hyaline bodies per se were not chemotactic for PMN, chemotactic activity was generated when hyaline bodies were incubated with serum. The mechanism by which chemotactic activity was generated, however, was not investigated. Recently, Kanagasundaram et al. (4) reported the presence of specific antibodies directed against hyaline bodies in serum from patients with acute alcoholic hepatitis. It is possible, therefore, that binding of these antibodies to

1. Abbreviations used in this paper: diHETE, dihydroxy-eicosatetraenoic acid; HPLC, high performance liquid chromatography; HETE, hydroxyeicosatetraenoic acid; FMLP, $\boldsymbol{N}$-formyl-methionyl-leucyl-phenylalanine; PMN, polymorphonuclear leukocyte(s); TLC, thin-layer chromatography. 
hyaline results in activation of complement and generation of complement-derived chemotactic peptides. More recently, Peters et al. (5) reported that incubation of human monocytes with highly purified Mallory bodies results in the generation of a factor that is chemotactic for PMN.

Of the studies that have been performed to date, none has addressed the possibility that chemoattractants may be derived directly from liver cells exposed to ethanol. In this report, we present evidence that ethanol stimulates isolated rat hepatocytes to produce a chemotactic factor for PMN.

\section{Methods}

Isolation and culture of rat hepatocytes. Normal rat hepatocytes were isolated and cultured as described previously (6). Briefly, livers of anesthetized Sprague-Dawley rats (young males weighing 100-150 g) were perfused in situ with calcium-free minimal essential medium (Gibco Laboratories, Grand Island, NY). After $5 \mathrm{~min}, 0.04 \%$ (wt/vol) bacterial collagenase (type I, Sigma Chemical Co., St. Louis, MO) and $2.0 \mathrm{mM} \mathrm{CaCl} 2$ were added to the perfusate. After an additional 25 min of perfusion, the markedly softened liver was removed and transferred to a flask that contained minimal essential medium, $0.04 \%$ (wt/vol) bacterial collagenase, $2.0 \mathrm{mM} \mathrm{CaCl}_{2}$, and $10 \mu \mathrm{g} / \mathrm{ml} \mathrm{DNase-1}$ (Sigma Chemical Co.). After opening the liver capsule with scissors, the flask was placed on a rotatory shaker for $5 \mathrm{~min}$ at $37^{\circ} \mathrm{C}$ to disperse enzymatically dissociated cells. Crude cell suspensions were subjected to centrifugal elutriation as described previously (7), using a Beckman JE-6B continuous flow rotor (Beckman Instruments, Inc., Palo Alto, CA). Subpopulations of cells banded in the elutriation chamber were removed sequentially by increasing the flow rate from the external pump while maintaining the rotor speed constant. Cell fractions that contained $>99 \%$ hepatic parenchymal cells were concentrated by centrifugation and resuspended in Medium 199 (Gibco Laboratories) that contained $1.0 \%$ (vol/vol) normal rat serum. Hepatocytes $\left(2 \times 10^{6}\right.$ cells) were plated onto plastic ( $35 \mathrm{~mm}$ diameter) Petri dishes (LUX Scientific, Inc., Newbury Park, CA) that had been coated previously with rat tail-tendon collagen (type I, Sigma Chemical Co.) (6). Cells were cultured at $37^{\circ} \mathrm{C}$ in an atmosphere of $5.0 \% \mathrm{CO}_{2}$ and $100 \%$ humidity for $24 \mathrm{~h}$ (during which viable cells became adherent). Plates were then washed twice with Medium 199 to remove nonadherent cells and subsequently cultured in fresh Medium 199 that contained $1.0 \%(\mathrm{vol} / \mathrm{vol})$ rat serum and experimental reagents. Hepatocyte viability was assessed by measuring trypan blue exclusion (6) and release into the culture medium of the cytoplasmic enzyme, lactate dehydrogenase (8). Lactate dehydrogenase was measured as described previously (8, 9) in cell-free culture supernatants and in supernatants after the addition of $0.2 \%$ (vol/vol) Nonidet-P40 (Sigma Chemical Co.) (total activity). Viability of hepatocytes usually exceeded $90 \%$ (by trypan blue exclusion) and was not influenced by any of the compounds that were added to the cultures. For some experiments, hepatocytes were cultured in Medium 199 that lacked rat serum. This did not alter the viability of the cultured cells.

Other cell cultures. Hepatic sinusoidal endothelial cells and Kupffer cells also were isolated from collagenase-treated rat livers by centrifugal elutriation, as described previously (10). Primary cultures of endothelial cells were $90-95 \%$ pure, with the principal contaminants being Kupffer cells (10). Primary cultures of Kupffer cells were $70-75 \%$ pure, with endothelial cells as the major contaminants $(7,11)$. Dermal fibroblasts
(95-99\% pure) were grown from explants of rat skin, as described previously (12), and used in their third passage.

Ethanol metabolism by hepatocytes. Metabolism of ${ }^{14} \mathrm{C}$-ethanol by hepatocytes was measured as ${ }^{14} \mathrm{C}$-acetate generation using the method of Grunnet et al. (13). Hepatocytes were plated onto $35-\mathrm{mm}$ plastic Petri dishes as described above. After $24 \mathrm{~h}$, cells were preincubated with either fresh medium alone or medium that contained varying concentrations of 4-methylpyrazole (Sigma Chemical Co.), an inhibitor of alcohol dehydrogenase (14), or cyanamide (Sigma Chemical Co.), an acetaldehyde dehydrogenase inhibitor (15). After $1 \mathrm{~h}$, the medium was replaced with fresh medium that contained $10 \mathrm{mM}$ unlabeled ethanol and $\sim 100,000 \mathrm{dpm} 1-^{14} \mathrm{C}$-ethanol $(53.4 \mathrm{mCi} / \mathrm{mmol}$, specific activity) (Amersham Corp., Arlington Heights, IL) with or without inhibitor. The cells were incubated at $37^{\circ} \mathrm{C}$ in sealed 50 - $\mathrm{ml}$ beakers on a rocking platform. After $6 \mathrm{~h}, 0.15 \mathrm{ml}$ of the culture medium was removed for assays of chemotactic activity, and the incubations were terminated by adding $0.4 \mathrm{ml}$ of $20 \%$ (vol/vol) trichloroacetic acid (Sigma Chemical Co.) to each culture dish. After solubilizing precipitated material with $0.3 \mathrm{ml}$ of $2.0 \mathrm{~N} \mathrm{NaOH}$ and brief sonication, aliquots $(0.4 \mathrm{ml})$ were transferred to glass scintillation vials and evaporated overnight at room temperature under vacuum in a desiccator that contained phosphorus pentoxide (J. T. Baker Chemical Co., Phillipsburg, $\mathrm{NJ})$. This was repeated twice with fresh phosphorus pentoxide and the residue was dissolved in $1.0 \mathrm{ml}$ glacial acetic acid and $10 \mathrm{ml}$ Aquasol (New England Nuclear Corp., Boston, MA). Blank samples contained no cells. Samples were dark-adapted for $24 \mathrm{~h}$ and counted with an efficiency of 93\% in a Packard Tri-Carb 460-C liquid scintillation counter (Packard Instrument Co., Inc., Downers Grove, IL).

Preparation of leukocyte suspensions. Venous blood $(32 \mathrm{ml})$ from healthy adult volunteers was allowed to sediment at room temperature after mixing with $8.0 \mathrm{ml}$ acid-citrate dextrose (National Institutes of Health formula A, Bethesda, MD) and $20 \mathrm{ml} \mathrm{6.0 \%} \mathrm{dextran} \mathrm{(average}$ molecular weight 234,000) (Sigma Chemical Co.) in $140 \mathrm{mM} \mathrm{NaCl}$. After adding $35 \mathrm{ml}$ of $\mathrm{NH}_{4} \mathrm{Cl}(8.7 \mathrm{mg} / \mathrm{ml})$ to $15-\mathrm{ml}$ aliquots of the leukocyte-rich supernatants, the mixtures were centrifuged at $155 \mathrm{~g}$ for $10 \mathrm{~min}$. Cell pellets were washed once with $140 \mathrm{mM} \mathrm{NaCl}$ and finally suspended in phosphate (10 mM)-buffered $140 \mathrm{mM} \mathrm{NaCl}, \mathrm{pH}$ 7.4 , supplemented with $0.6 \mathrm{mM} \mathrm{CaCl}_{2}, 1.0 \mathrm{mM} \mathrm{MgCl}$ and $20 \mathrm{mg} /$ $\mathrm{ml}$ bovine serum albumin (Gibco Laboratories). This buffer was used throughout. Cell suspensions prepared in this fashion contained $\sim 85 \%$ PMN.

PMN chemotaxis. Directed migration (chemotaxis) of PMN was assayed using a minor modification (9) of the "leading front" method of Zigmond and Hirsch (16). Stimulated random motility (chemokinesis) in response to albumin-containing buffer and total migration in response to chemoattractants are reported as the distance $(\mu \mathrm{m} / 35 \mathrm{~min})$ that the leading front of cells migrated into $3.0-\mu \mathrm{m}$ pore-diameter cellulose nitrate filters (Sartorious Filters, Inc., Hayward, CA) that separated the upper, or cell compartments, from the lower, or stimulus compartments, of modified Boyden chambers (Nuclepore Corp., Pleasanton, CA). Chemotaxis (net migration) was calculated by subtracting stimulated random motility from total migration. Duplicate chambers were used in each experiment and 10 fields were examined in each filter. The synthetic chemotactic factor, $N$-formyl-methionyl-leucylphenylalanine (FMLP), was obtained from Peninsula Laboratories, Inc., San Carlos, CA.

In some experiments, the method described by Maderazo and Woronick (17) was used to assess the migratory behavior of the entire population of PMN. A leukotactic index, which reflects the mean distance traveled by all cells, was determined by counting the number 
of PMN at various depths (10- $\mu \mathrm{m}$ intervals) in the micropore filters using an image analyzer (Optomax Inc., Hollis, $\mathrm{NH}$ ) interfaced with an Apple II computer (Apple Computer Inc., Cupertino, CA).

Lipid extraction and thin-layer chromatography (TLC). Supernatants $(20 \mathrm{ml})$ from hepatocytes that had been cultured for $6 \mathrm{~h}$ in the presence and absence of $10 \mathrm{mM}$ ethanol were acidified to $\mathrm{pH} 4.0$ with $6 \mathrm{~N} \mathrm{HCl}$ and extracted by adding $20 \mathrm{vol}$ of chloroform/methanol (2:1). After vigorous mixing, 4 vol of distilled water was added and the resulting organic and aqueous phases were isolated.

TLC was performed on silica gel 60 plates (Brinkmann Instruments Inc., Westbury, NY) which had been washed with methanol and activated for $30 \mathrm{~min}$ at $110^{\circ} \mathrm{C}$. Samples were applied under nitrogen and chromatography was performed using a solvent system that consisted of ethyl acetate/isooctane/glacial acetic acid/water (90:50:20:100). Plates were dried under nitrogen and either scraped or scanned for radioactivity using a Berthold TLC-scanner (Berthold Instruments, Inc., Pittsburgh, PA). Scraped sections were extracted with chloroform/methanol (1:1), and the organic phase evaporated under nitrogen. Extracted material was dissolved in absolute ethanol and aliquots diluted in buffer that contained albumin for chemotaxis assays.

High-performance liquid chromatography (HPLC). Reverse-phase HPLC was performed as described previously (18), using a $4.6 \times 250$ mm Ultrasphere ODS column (Beckman Instruments, Inc.) and a mobile phase that consisted of methanol/water/acetic acid (79:21:0.01). The column was eluted at a rate of $1.0 \mathrm{ml} / \mathrm{min}$ with continuous monitoring for ultraviolet absorbance at $280 \mathrm{~nm}$.

Other reagents. ${ }^{3} \mathrm{H}$-arachidonic acid $(87.4 \mathrm{Ci} / \mathrm{mmol})$ was obtained from New England Nuclear. ${ }^{3} \mathrm{H}$-leukotriene $\mathbf{B}_{4}$ (5,12-dihydroxy6,8,10,14-eicosatetraenoic acid) and ${ }^{3} \mathrm{H}-5(S)$-hydroxy-eicosatetraenoic acid (5-HETE) were prepared by stimulating human PMN $\left(50 \times 10^{6}\right)$ $\mathrm{ml})$ with the calcium ionophore, A23187 $(10 \mu \mathrm{m})$ (Sigma Chemical Co. $)$ in the presence of ${ }^{3} \mathrm{H}$-arachidonic acid $(10 \mu \mathrm{Ci} / \mathrm{ml})$, and was purified by reverse-phase HPLC (18). Radiolabeled 15-HETE and $8(S), 15(S)$-dihydroxy-5,11-cis-9,13-trans-eicosatetraenoic acid $(8,15$ diHETE) were prepared using soybean lipoxygenase (18). Human platelets were used to generate radiolabeled 12-HETE and 12(S)hydroxy-heptadecatrienoic acid (19). The epoxides, 5,6-, 8,9-, 11,12-, and 13,14-oxido-eicosatrienoic acids, as well as the corresponding vicinal diols, were synthesized from arachidonic acid (NuChek Prep, Elysian, MN) using $m$-chloroperoxybenzoic acid (Aldrich Chemical Co., Inc., Milwaukee, WI), and were purified by HPLC as described by Oliw and Moldens (20). Radiolabeled thromboxane $B_{2}$ and prostaglandin $E_{2}$ were obtained from New England Nuclear. Superoxide dismutase and catalase were obtained from Sigma Chemical Co.

\section{Results}

Generation of chemotactic activity by rat hepatocytes. Media from individual dishes of cultured rat hepatocytes were harvested and centrifuged briefly to remove any detached cells. The resulting supernatants were diluted and assayed for chemotactic activity. The results of experiments in which supernatants from 18-h cultures of rat hepatocytes were tested for their ability to stimulate migration of human PMN are shown in Table I. Supernatants $(0.5 \%$, vol/vol $)$ from cultures without ethanol failed to stimulate PMN migration beyond that seen with albumin-containing buffer. In contrast, significantly en-
Table I. Supernatants of Rat Hepatocytes Cultured for $18 \mathrm{~h}$ with Ethanol Stimulate Migration of Human PMN

\begin{tabular}{ll}
\hline Stimulus & PMN migration \\
\hline & $\mu \mathrm{m} / 35 \mathrm{~min}^{*}$ \\
Buffer (stimulated random migration) & $83.3 \pm 2.1$ \\
Culture supernatants $(0.5 \%, \mathrm{vol} / \mathrm{vol})$ & \\
Hepatocytes alone & $81.9 \pm 1.6$ \\
Hepatocytes plus ethanol $(10 \mathrm{mM})$ & $97.3 \pm 1.9 \ddagger$ \\
\hline
\end{tabular}

* Results represent mean values $( \pm \mathrm{SE})$ from six experiments that were performed with different preparations of both hepatocytes and PMN.

$\ddagger P$ vs. buffer and hepatocytes alone $<0.01$ ( $t$ test).

hanced migration of PMN was observed in response to supernatants $(0.5 \%, \mathrm{vol} / \mathrm{vol})$ of hepatocytes that were cultured for $18 \mathrm{~h}$ with $10 \mathrm{mM}$ ethanol.

Since the "leading front" method of assaying chemotaxis measures only the distance traveled by the fastest migrating cells, PMN responses to culture supernatants were also examined using the method of Maderazo and Woronick (17). In a representative experiment, leukotactic indices calculated for PMN responses to albumin-containing buffer, culture supernatants $(0.5 \%, \mathrm{vol} / \mathrm{vol})$ of untreated hepatocytes, and culture supernatants $(0.5 \%$, vol/vol) of ethanol $(10 \mathrm{mM})$-treated hepatocytes were $8.3,9.1$, and 15.8 , respectively.

By varying the concentration of stimulus above and below the filter in the Boyden chamber assay, stimulated random migration of PMN (or chemokinesis) was distinguished from true directed migration (or chemotaxis) (16). Results of such a "checkerboard" analysis are shown in Table II. When culture supernatants from ethanol-treated hepatocytes (10 mM, $18 \mathrm{~h}$ ) were placed only above the filters, a concentration of $0.3 \%$

Table II. Analysis of Chemokinetic and

Chemotactic Activities in Supernatants of Rat Hepatocytes Cultured for $18 \mathrm{~h}$ with $10 \mathrm{mM}$ Ethanol

\begin{tabular}{lllll}
\hline & \multicolumn{4}{l}{ Culture supernatants above filter $(\%, v o l / v o l)$} \\
\cline { 2 - 5 } $\begin{array}{l}\text { Culture supernatants } \\
\text { below filter }\end{array}$ & 0.0 & 0.1 & 0.3 & 0.5 \\
\hline \%, vol/vol & & & & \\
0.0 & 74.6 & 75.4 & 78.0 & 73.8 \\
0.1 & 78.8 & 73.7 & 77.9 & 73.0 \\
0.3 & 85.6 & 80.2 & 79.9 & 74.3 \\
0.5 & 90.5 & 84.9 & 80.2 & 74.2
\end{tabular}

Results are expressed as PMN migration ( $\mu \mathrm{m} / 35 \mathrm{~min})$ toward either buffer alone or varying concentrations of culture supernatants added to the cell compartments (above filter) and/or to the stimulus compartments (below filter) of modified Boyden chambers. Values represent the average of two experiments performed with different preparations of both hepatocytes and PMN 
(vol/vol) stimulated random migration of PMN. Some stimulated random migration (or chemokinesis) also was observed when supernatants were added simultaneously above and below the filters at a concentration of $0.3 \%$ ( $\mathrm{vol} / \mathrm{vol}$ ). In contrast, when cells were exposed to positive gradients of active supernatants, that is, higher concentrations below the filters, they responded by migrating in a directed fashion. Thus, culture supernatants from ethanol-treated hepatocytes were truly chemotactic and also exhibited chemokinetic activity at low concentrations.

Responses of PMN to varying concentrations of supernatants from rat hepatocytes that were cultured with $10 \mathrm{mM}$ ethanol for $18 \mathrm{~h}$ are shown in Fig. 1. Significant net migration of PMN was observed in response to culture supernatants that were tested at a concentration of $0.3 \%$ ( $\mathrm{vol} / \mathrm{vol})$. Maximum net migration of PMN was observed in response to $0.5 \%$ of the culture supernatants. Higher concentrations caused decreased PMN migration, a phenomenon observed with other potent chemoattractants and termed "deactivation" (21).

Generation of chemotactic activity by ethanol-treated rat hepatocytes varied with the concentration of ethanol and with the duration of incubation (Fig. 2). Fig. $2 A$ illustrates the responses of $\mathrm{PMN}$ to supernatants $(0.5 \%$, vol/vol) of rat hepatocytes that were cultured for $18 \mathrm{~h}$ with varying concentrations of ethanol. Significant amounts of chemotactic activity were detected in supernatants of hepatocytes that were cultured with concentrations of ethanol as low as $2.0 \mathrm{mM}$. Maximum amounts of chemotactic activity were recovered from cells cultured with $10 \mathrm{mM}$ ethanol. Concentrations of ethanol as high as $50 \mathrm{mM}$ did not induce further generation of chemotactic activity (not shown). It should be noted that during the incubation periods (with and without ethanol), hepatocytes remained $>90 \%$ viable, as assessed by trypan blue exclusion, and released $<3.0 \%$ of total lactate dehydrogenase activity.

Shown in Fig. $2 B$ are the responses of PMN to supernatants $(0.5 \%, \mathrm{vol} / \mathrm{vol})$ of rat hepatocytes that were cultured with 10 $\mathrm{mM}$ ethanol for up to $20 \mathrm{~h}$. Significant chemotactic activity was detected after $2 \mathrm{~h}$ of incubation and was maximal after 6 h. Chemotactic activity diminished with more prolonged incubations. This phenomenon was observed consistently and was not due simply to PMN deactivation by high concentrations

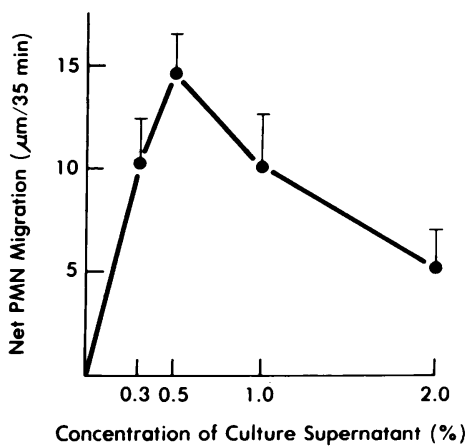

Figure 1. Responses of PMN to varying concentrations of supernatants that were recovered from rat hepatocytes cultured for $18 \mathrm{~h}$ with ethanol $(10 \mathrm{mM})$. Results are expressed as net PMN migration $(\mu \mathrm{m} / 35$ $\min$ ), and were calculated by subtracting random migration in response to buffer alone from total migration (mean $\pm \mathrm{SE}, n=3$ ).

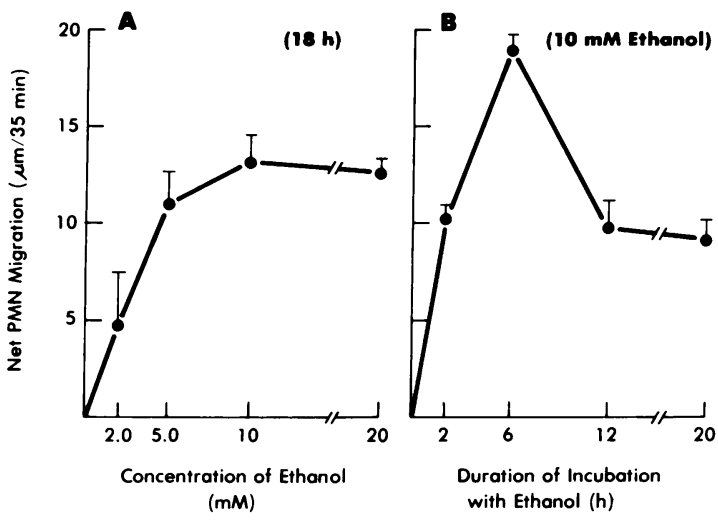

Figure 2. Generation of chemotactic activity by ethanol-treated rat hepatocytes varies with the concentration of ethanol and duration of incubation. In $A$, hepatocytes were cultured for $18 \mathrm{~h}$ with $0-20 \mathrm{mM}$ ethanol. In $B$, hepatocytes were cultured with $10 \mathrm{mM}$ ethanol for 0 $20 \mathrm{~h}$. Results represent net PMN migration in response to $0.5 \%$ (vol/ vol) culture supernatants (mean $\pm \mathrm{SE}, n=3$ ).

of chemoattractants. Further dilutions of the 20-h supernatants did not result in enhanced PMN migration (not shown).

The ability of cell types other than hepatic parenchymal cells to generate chemotactic activity when incubated with ethanol also was examined. In contrast to the results obtained with ethanol-treated rat hepatocytes, ethanol $(10 \mathrm{mM})$-treated rat dermal fibroblasts, hepatic sinusoidal endothelial cells, and Kupffer cells failed to generate detectable chemotactic activity (data not shown). Thus, cells that potentially may contaminate cultures of rat hepatocytes were not a source of the observed chemotactic activity.

Ethanol metabolism by rat hepatocytes is required for the generation of chemotactic activity. Ethanol is converted to acetaldehyde in rat (and human) hepatocytes by the enzyme alcohol dehydrogenase (22). Acetaldehyde is metabolized further to acetate either by acetaldehyde dehydrogenase or by an acetaldehyde oxidase system $(22,23)$. In order to determine whether the generation of chemotactic activity by hepatocytes required metabolism of ethanol, experiments were performed using ${ }^{14} \mathrm{C}$-ethanol and the alcohol dehydrogenase inhibitor, 4methylpyrazole (14). These experiments demonstrated a close relationship between ethanol metabolism by rat hepatocytes and generation of chemotactic activity (Fig. 3). Incubation of rat hepatocytes with varying concentrations of 4-methylpyrazole resulted in a dose-dependent inhibition of the generation of both ${ }^{14} \mathrm{C}$-acetate (as a measure of ethanol metabolism) and chemotactic activity. In experiments not shown, 4-methylpyrazole $(1.0 \mathrm{mM})$ did not influence stimulated random migration of PMN, nor did it impair the ability of PMN to respond chemotactically to the synthetic peptide, FMLP.

To determine whether generation of chemotactic activity by hepatocytes required metabolism of acetaldehyde, experiments were performed with the acetaldehyde dehydrogenase 


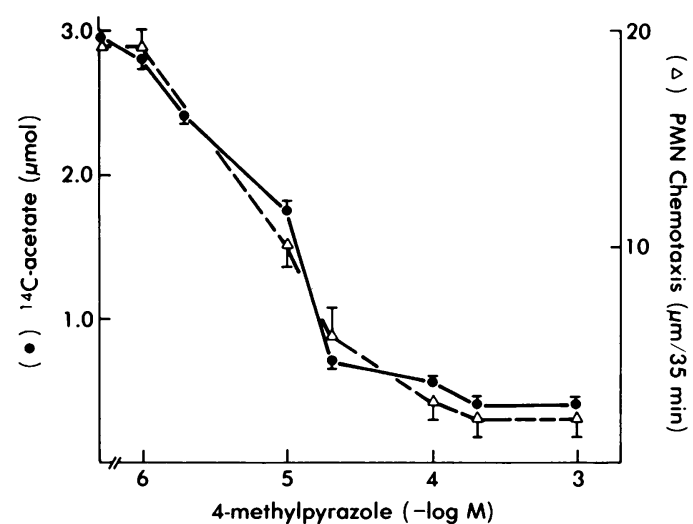

Figure 3. Metabolism of ethanol and generation of chemotactic activity by hepatocytes is inhibited by 4-methylpyrazole. Hepatocytes were cultured for $6 \mathrm{~h}$ with $10 \mathrm{mM}$ unlabeled ethanol, $100,000 \mathrm{dpm}{ }^{14} \mathrm{C}$ ethanol, and varying concentrations of 4-methylpyrazole. Ethanol metabolism was assessed by measuring generation of ${ }^{14} \mathrm{C}$-acetate $\left(\mu \mathrm{mol} / 2 \times 10^{6}\right.$ hepatocytes $\left./ 6 \mathrm{~h}\right)$. Chemotactic activity was measured as net PMN migration toward $0.5 \%$ (vol/vol) culture supernatants (average of two experiments).

inhibitor, cyanamide (15). As shown in Table III, $1.0 \mathrm{mM}$ cyanamide completely inhibited the generation of chemotactic activity by hepatocytes that were cultured with $10 \mathrm{mM}$ ethanol. It should be noted that $1.0 \mathrm{mM}$ cyanamide almost completely inhibits generation of ${ }^{14} \mathrm{C}$-acetate from ${ }^{14} \mathrm{C}$-ethanol (15), but does not alter hepatocyte viability. As was the case with 4-

Table III. Effects of Cyanamide, Superoxide Dismutase, and Catalase on the Generation of Chemotactic Activity by Rat Hepatocytes Cultured for $6 \mathrm{~h}$ with $10 \mathrm{mM}$ Ethanol

\begin{tabular}{ll}
\hline Stimulus & PMN migration \\
\hline Buffer & $\mu \mathrm{m} / 35 \mathrm{~min}^{*}$ \\
Culture supernatants $(0.5 \%$, vol/vol) & 80.8 \\
$\quad$ Hepatocytes alone & 81.9 \\
$\quad$ Hepatocytes plus cyanamide $(1.0 \mathrm{mM})$ & 82.8 \\
Hepatocytes plus ethanol $(10 \mathrm{mM})$ & 99.5 \\
$\quad$ Hepatocytes plus cyanamide $(1.0 \mathrm{mM})$ & \\
$\quad$ plus ethanol $(10 \mathrm{mM}) \ddagger$ & 81.3 \\
$\quad$ Hepatocytes plus ethanol $(10 \mathrm{mM})$ plus & \\
$\quad$ cyanamide $(1.0 \mathrm{mM}) \S$ & 96.4 \\
Hepatocytes plus superoxide dismutase & \\
$\quad(100 \mu \mathrm{g} / \mathrm{ml})$ plus catalase $(100 \mu \mathrm{g} / \mathrm{ml})$ & \\
$\quad$ plus ethanol $(10 \mathrm{mM})$ & 102.8 \\
\hline
\end{tabular}

* Results represent average values from two experiments performed with different preparations of both hepatocytes and PMN. ¥ Cyanamide was added just before the addition of ethanol. $\S$ Cyanamide was added after hepatocytes were incubated with ethanol for $6 \mathrm{~h}$. methylpyrazole, cyanamide did not influence either random migration of PMN or chemotactic responses of PMN to FMLP (data not shown). Addition of cyanamide to culture supernatants from ethanol-treated hepatocytes did not affect the ability of the supernatants to stimulate PMN directed migration (Table III). Furthermore, incubation of rat hepatocytes for 6 $h$ with varying concentrations of acetaldehyde $\left(10^{-6}-10^{-3} \mathrm{M}\right)$ resulted in a dose-dependent generation of chemotactic activity for PMN (data not shown). Generation of chemotactic activity by acetaldehyde-treated rat hepatocytes was inhibited almost completely by $1.0 \mathrm{mM}$ cyanamide. Thus, metabolism of both ethanol and acetaldehyde were required for the generation of chemotactic activity by ethanol-treated hepatocytes.

Since metabolism of acetaldehyde by hepatocytes may lead to the generation of superoxide anion radicals and hydrogen peroxide (23), we examined whether these oxygen metabolites were involved in the production of chemotactic activity by ethanol-treated cells. As shown in Table III, hepatocytes that were incubated with $10 \mathrm{mM}$ ethanol in the presence of 100 $\mu \mathrm{g} / \mathrm{ml}$ superoxide dismutase and $100 \mu \mathrm{g} / \mathrm{ml}$ catalase generated as much chemotactic activity as hepatocytes that were incubated with ethanol alone.

Partial characterization of the chemotactic activity generated by ethanol-treated hepatocytes. Although the medium routinely used to culture hepatocytes contained $1.0 \%$ (vol/vol) rat serum, generation of chemotactic activity by ethanol-treated hepatocytes was not dependent upon the presence of serum. Results identical to those presented above were obtained when cells were cultured in the absence of serum (not shown).

In preliminary efforts to isolate and characterize the chemotactic activity that was generated by ethanol-treated hepatocytes, culture supernatants were extracted with chloroform/ methanol. Chloroform/methanol extracts of culture supernatants contained (in the chloroform phase) $>90 \%$ of the chemotactic activity that was generated when hepatocytes were incubated with $10 \mathrm{mM}$ ethanol for $6 \mathrm{~h}$ (not shown). No chemotactic activity was detected in the organic (or aqueous) phases of extracts that were prepared from supernatants of hepatocytes cultured in medium without ethanol. These findings suggested that the chemotactic factor produced by ethanoltreated hepatocytes was relatively nonpolar and, therefore, likely to be a lipid.

Since a number of arachidonic acid metabolites possess potent chemotactic activity for $\operatorname{PMN}(18,19,24)$, we investigated whether the chemotactic factor produced by ethanoltreated hepatocytes might be derived from arachidonic acid. Chloroform/methanol extracts of supernatants from ethanoltreated hepatocytes were subjected to TLC on silica gel 60 plates. A mixture of tritiated eicosanoid standards were run in a parallel lane. After chromatography, plates were scanned for radioactivity and scraped so that fractions could be tested for chemotactic activity. As shown in Fig. 4, chemotactic activity was detected only in a fraction that co-migrated with the dihydroxylated derivatives of arachidonic acid, leukotriene $\mathbf{B}_{\mathbf{4}}$, 


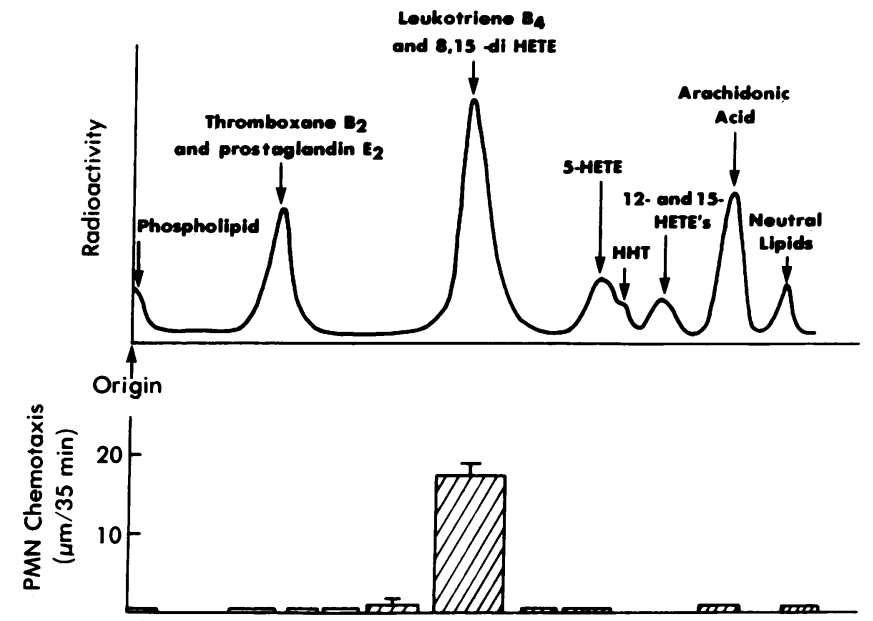

Figure 4. Analysis by TLC of the chemotactic activity for PMN generated by ethanol-treated hepatocytes. The tracing (top) was obtained after chromatography of a mixture of authentic, ${ }^{3} \mathrm{H}$-labeled standards. Shown below is the chemotactic activity recovered by scraping sections of the plate after TLC of a supernatant (chloroform/methanol extract) from hepatocytes that had been incubated with $10 \mathrm{mM}$ ethanol for $6 \mathrm{~h}$.

and 8,15-diHETE. Similar results were obtained when chloroform/methanol extracts were subjected to analysis by HPLC. Chemotactic activity was detected only in a fraction that corresponded to a peak (absorbance at $280 \mathrm{~nm}$ ) that exhibited a retention time $(\sim 11 \mathrm{~min})$ similar to that observed when authentic leukotriene $B_{4}$ was chromatographed under identical conditions. Thus, the chemotactic factor generated by ethanoltreated rat hepatocytes exhibited properties on TLC and HPLC similar to those of other chemotactic, moderately polar lipids.

In order to determine directly whether the chemotactic factor was derived from arachidonic acid, hepatocytes were incubated with ${ }^{3} \mathrm{H}$-arachidonic acid for $18 \mathrm{~h}\left(1.0 \mu \mathrm{Ci} / 4 \times 10^{6}\right.$ cells). Analysis of cell extracts by TLC revealed that the radiolabeled arachidonic acid was incorporated efficiently. Approximately $85 \%$ of the radioactivity was detected at the origin (co-migrating with phospholipid standards) and could be recovered as unaltered arachidonic acid after base hydrolysis. The remaining radioactivity was recovered in fractions that co-migrated with neutral lipid standards. After incubation of the prelabeled hepatocytes with ethanol $(10 \mathrm{mM}, 6 \mathrm{~h})$, analysis by TLC of material extracted from supernatants with chloroform/methanol failed to reveal significant amounts of radioactivity associated with the peak of chemotactic activity (not shown). Analysis by HPLC also failed to reveal any significant amounts of radioactivity (or ultraviolet absorbance) that cochromatographed with known chemotactic products of arachidonic acid, such as leukotriene $B_{4}, 8,15$-diHETE, or 5-HETE. Thus, we were unable to confirm that ethanol-treated hepatocytes convert exogenous, radiolabeled arachidonic acid to a known product with chemotactic activity. It is possible, how- ever, that exogenous, radiolabeled arachidonic acid failed to enter an endogenous pool in rat hepatocytes that was available for conversion to biologically active products.

It has been demonstrated recently (20) that arachidonic acid can be converted as a result of cytochrome P-450mediated epoxidation by isolated rat hepatic microsomes and intact rat hepatocytes to several epoxides, as well as vicinal diols (e.g., 5,6-, 8,9-, 11,12-, 14,15-oxido- and diHETEs). We synthesized these compounds and examined whether they might be related to the chemotactic factor generated by ethanoltreated hepatocytes. None of the synthetic epoxides or vicinal diols exhibited significant chemotactic activity for human PMN when tested at concentrations ranging from 0.1 to 1.0 $\mu \mathrm{M}$ (not shown). Thus, it is unlikely that these compounds account for the chemotactic activity generated by ethanoltreated rat hepatocytes.

\section{Discussion}

Results of the studies described in this report indicate that ethanol stimulates cultured rat hepatocytes to generate chemotactic activity for human PMN (Tables I and II, Fig. 1). Generation of chemotactic activity varied with the concentration of ethanol (Fig. $2 A$ ) and with the duration of incubation (Fig. $2 \mathrm{~B}$ ). Maximal amounts of activity were recovered from culture supernatants after $6 \mathrm{~h}$ of incubation with $10 \mathrm{mM}$ ethanol. Incubation of hepatocytes with higher concentrations of ethanol did not result in the appearance of more chemotactic activity. This is not surprising since the generation of chemotactic activity is closely linked to the metabolism of ethanol (Fig. 3), and the $K_{\mathrm{m}}$ of alcohol dehydrogenase in rat hepatocytes is $\sim 2.0-4.0 \mathrm{mM}(22)$. Supernatants from cells incubated with $10 \mathrm{mM}$ ethanol for longer than $6 \mathrm{~h}$ consistently contained less chemotactic activity, which suggested that the chemotactic factor was either altered (e.g., oxidized) during prolonged incubations or metabolized further by the hepatocytes.

Generation of chemotactic activity by rat hepatocytes required metabolism of ethanol by the alcohol dehydrogenase system (Fig. 3) and the acetaldehyde dehydrogenase system (Table III). Inhibition of the former by 4-methylpyrazole or the latter by cyanamide abrogated the production of chemotactic activity by hepatocytes incubated with ethanol. Furthermore, hepatocytes that were incubated with acetaldehyde alone generated significant amounts of chemotactic activity. These observations argue against either a nonspecific or a toxic effect of ethanol on the cells as a mechanism for the generation of chemotactic activity. Our inability to detect chemotactic activity in cultures of several nonparenchymal cell types is consistent with previous studies which have shown that, in contrast to hepatocytes, these cells do not metabolize ethanol (25).

The chemotactic activity in supernatants of ethanol-treated hepatocytes was recovered almost completely by extraction with chloroform/methanol, and cochromatographed on TLC (Fig. 4) and HPLC (not shown) with dihydroxylated derivatives 
of arachidonic acid (specifically leukotriene $B_{4}$ ). The chromatographic behavior was quite different, however, from that observed in experiments that were performed with the chemotactic phospholipid, platelet-activating factor (26). Although these results suggest that the chemotactic factor is a relatively polar lipid, they do not exclude the possibility that it is a low molecular weight hydrophobic peptide (e.g., an $\mathrm{N}$-formylated peptide). Furthermore, our attempts to demonstrate that ethanol-treated hepatocytes directly convert radiolabeled arachidonic acid to known biologically active products were unsuccessful. The possibility remains, however, that small amounts of chemotactically active products are generated by ethanol-treated rat hepatocytes from an endogenous pool of arachidonic acid that is not exchangeable with exogenous ${ }^{3} \mathrm{H}$ arachidonic acid. This possibility is supported by results of preliminary experiments in which supernatants of cultured hepatocytes were examined for the presence of leukotriene $\mathbf{B}_{4}$ using a specific radioimmunoassay (27) (kindly performed by Dr. Edward J. Goetzl, University of California, San Francisco). Supernatants of hepatocytes that had been cultured for $6 \mathrm{~h}$ with $10 \mathrm{mM}$ ethanol contained $6.7 \mathrm{pmol} / \mathrm{ml}$ of immunoreactive leukotriene $B_{4}$, while supernatants of hepatocytes that were cultured with ethanol plus $1.0 \mathrm{mM}$ 4-methylpyrazole contained only $2.2 \mathrm{pmol} / \mathrm{ml}$ of immunoreactive leukotriene $B_{4}$.

Alternative mechanisms for the generation by ethanoltreated hepatocytes of a chemotactic lipid need to be considered. Ethanol is oxidized in the liver to acetaldehyde (by alcohol dehydrogenase) and then to acetate (by either acetaldehyde dehydrogenase or acetaldehyde oxidase) $(22,23)$. The latter enzyme is "linked" to an NADPH-cytochrome $P-450$ reductase system that may generate several potentially toxic oxygen metabolites (e.g., superoxide anion radicals, hydroxyl radicals, singlet oxygen) as byproducts of acetaldehyde oxidation (23, 28-30). Toxic oxygen metabolites may be involved in the formation of lipid peroxides that accompanies ethanol metabolism by hepatocytes, and have been implicated as mediators of ethanol-induced liver injury $(23,31,32)$. Oxygen-derived free radicals can also stimulate arachidonic acid metabolism (33) and can act directly on arachidonic acid (and perhaps other lipids) to yield products with chemotactic activity (34, 35). These observations are particularly relevant since we found that acetaldehyde alone stimulated rat hepatocytes to generate chemotactic activity. Nevertheless, we were unable to inhibit generation of chemotactic activity by ethanol-treated hepatocytes with high concentrations of superoxide dismutase and catalase (Table III).

Our current efforts are directed at identifying more precisely the chemotactic factor that is generated by ethanol-treated rat hepatocytes, characterizing its mechanism of production, and demonstrating that human, as well as rat hepatocytes respond to ethanol by generating chemotactic activity for PMN. The findings reported here strongly suggest that ethanol-induced generation by hepatocytes of chemoattractants may account, in part, for the PMN infiltrates that are characteristically found in the liver parenchyma early in the course of acute alcoholic hepatitis.

\section{Acknowledgments}

The authors thank Dr. Edward J. Goetzl, University of California, San Francisco, for his help in performing radioimmunoassays of leukotriene $B_{4}$.

This work was aided by grants from the National Institutes of Health (AM-28566, HL-28475, AM-26743, AM-00919, AM-31198, and AM-26743), the Treadwell Foundation, and the Academic Senate Committee on Research, University of California, San Francisco. Dr. Shak was supported by a National Pulmonary Faculty Training Center grant (HL-07159) from the National Institutes of Health.

\section{References}

1. French, S. W., and E. J. Burbige. 1979. Alcoholic hepatitis: clinical, morphologic, pathogenetic and therapeutic aspects. Prog. Liver Dis. 6:557-579.

2. Boitnott, J. K., and W. C. Maddrey. 1981. Alcoholic liver disease: I. Interrelationships among histologic features and the histologic effects of prednisolone therapy. Hepatology (Balt.). 1:599-612.

3. Leevy, C. M., T. Chen, A. Luisada-Opper, N. Kanagasundaram, and $R$. Zetterman. 1976. Liver disease of the alcoholic: role of immunologic abnormalities in pathogenesis, recognition and treatment. Prog. Liver Dis. 5:516-530.

4. Kanagasundaram, N., S. Kakumu, T. Chen, and C. M. Leevy. 1977. Alcoholic hyaline antigen (AHAg) and antibody (AHAb) in alcoholic hepatitis. Gastroenterology. 73:1368-1373.

5. Peters, M., H. A. Liebman, M. J. Tong, and H. M. Tinberg. 1983. Alcoholic hepatitis: granulocyte chemotactic factor from Mallory body-stimulated human peripheral blood mononuclear cells. Clin. Immunol. Immunopathol. 28:418-430.

6. Bissell, D. M., and P. S. Guzelian. 1980. Phenotypic stability of adult rat hepatocytes in primary monolayer culture. Ann. NY Acad. Sci. 349:85-98.

7. Knook, D. L., and E. C. Sleyster. 1976. Separation of Kupffer and endothelial cells of the rat liver by centrifugal elutriation. Exp. Cell Res. 99:444-449.

8. Wacker, W. E. C., D. D. Ulmer, and B. L. Vallee. 1956 Metalloenzymes and myocardial infarction. II. Malic and lactic dehydrogenase activities and zinc concentration in serum. N. Engl. J. Med. 255:449-456.

9. Perez, H. D., M. Lipton, and I. M. Goldstein. 1978. A specific inhibitor of complement (C5)-derived chemotactic activity in serum from patients with systemic lupus erythematosus. J. Clin. Invest. 62:29-38.

10. Irving, M. G., F. J. Roll, and D. M. Bissell. 1981. Sinusoidal endothelial cells from normal rat liver: isolation, culture and collagen phenotype. Hepatology (Balt.). 1:520.

11. Mills, D. M., and D. Zucker-Franklin. 1969. Electron microscopic study of isolated Kupffer cells. Am. J. Pathol. 54:147-155.

12. Nadler, H. L., T. Inouye, P. Justice, and D. Y. Y. Hsia. 1967. Enzymes in cultivated human fibroblasts derived from patients with Down's syndrome (mongolism). Nature (Lond.). 213:1261-1262.

13. Grunnet, N., B. Quistorff, and H. I. D. Thieden. 1973. Ratelimiting factors in ethanol oxidation by isolated rat liver parenchymal cells. Eur. J. Biochem. 40:275-282. 
14. Theorell, H., T. Youstani, and B. Sjoberg. 1969. On the effects of some heterocyclic compounds on the enzymic activity of liver alcohol dehydrogenase. Acta Chem. Scand. 23:255-260.

15. Cederbaum, A. I. 1981. The effect of cyanamide on acetaldehyde oxidation by isolated rat liver mitochondria and on the inhibition of pyruvate oxidation by acetaldehyde. Alcohol. Clin. Exp. Res. 5:38-44.

16. Zigmond, S. H., and J. G. Hirsch. 1973. Leukocyte locomotion and chemotaxis: new methods for evaluation and demonstration of a cell-derived chemotactic factor. J. Exp. Med. 137:387-410.

17. Maderazo, E. G., and C. L. Woronick. 1978. Micropore filter assay of human granulocyte locomotion: problems and solutions. Clin. Immunol. Immunopathol. 11:196-211.

18. Shak, S., H. D. Perez, and I. M. Goldstein. 1983. A novel dioxygenation product of arachidonic acid possesses potent chemotactic activity for human polymorphonuclear leukocytes. J. Biol. Chem. 258:14948-14953.

19. Goetzl, E. J., and R. R. Gorman. 1978. Chemotactic and chemokinetic stimulation of human eosinophil and neutrophil polymorphonuclear leukocytes by 12-L-hydroxy-5,8,10-heptadecatrienoic acid (HHT). J. Immunol. 120:526-531.

20. Oliw, E. H., and P. Moldens. 1982. Metabolism of arachidonic acid by isolated rat hepatocytes, renal cells and by some rabbit tissues. Detection of vicinal diols by mass fragmentography. Biochim. Biophys. Acta. 721:135-143.

21. Ward, P. A., and E. L. Becker. 1968. The deactivation of rabbit neutrophils by chemotactic factor and the nature of the activatable esterase. J. Exp. Med. 127:693-709.

22. Li, T.-K. 1977. Enzymology of human alcohol metabolism. Adv. Enzymol. 45:427-483.

23. Lewis, K. O., and A. Paton. 1982. Could superoxide cause cirrhosis? Lancet. II:188-189.

24. Goetzl, E. J., and W. C. Pickett. 1980. The human PMN leukocyte chemotactic activity of complex hydroxy-eicosatetraenoic acids (HETEs). J. Immunol. 125:1789-1791.

25. Sjoblom, M., and J. Morland. 1979. Metabolism of ethanol and acetaldehyde in parenchymal and nonparenchymal rat liver cells. Biochem. Pharmacol. 28:3417-3423.

26. Demopoulos, C. A., R. N. Pinckard, and D. J. Hanahan. 1979. Platelet activating factor. Evidence for 1-O-alkyl-2-acetyl-sn-glyceryl3-phosphorylcholine as the active component (a new class of lipid chemical mediators). J. Biol. Chem. 254:9355-9358.

27. Holtzman, M. J., H. Aizawa, J. A. Nadel, and E. J. Goetzl. 1983. Selective generation of leukotriene $B_{4}$ by tracheal epithelial cells from dogs. Biochem. Biophys. Res. Commun. 114:1071-1076.

28. Cederbaum, A. I., E. Dicker, and G. Cohen. 1978. Effect of hydroxyl radical scavengers on microsomal oxidation of alcohols and on associated microsomal reactions. Biochemistry. 17:3058-3064.

29. Winston, G. W., and A. I. Cederbaum. 1983. NADPHdependent production of oxy radicals by purified components of the rat liver mixed function oxidase system. I. Oxidation of hydroxyl radical scavenging agents. J. Biol. Chem. 258:1508-1513.

30. Winston, G. W., and A. I. Cederbaum. 1983. NADPHdependent production of oxy radicals by purified components of the rat liver mixed function oxidase system. II. Role in microsomal oxidation of ethanol. J. Biol. Chem. 258:1514-1519.

31. DiLuzio, N. R. 1973. Antioxidants, lipid peroxidation and chemical-induced liver injury. Fed. Proc. 32:1875-1881.

32. Lieber, C. S. 1980. Metabolism and metabolic effects of alcohol. Semin. Hematol. 17:85-99.

33. Baud, L., M. P. Nivez, D. Chansel, and R. Ardaillou. 1981. Stimulation by oxygen radicals of prostaglandin production by rat renal glomeruli. Kidney Int. 20:332-339.

34. Perez, H. D., B. B. Weksler, and I. M. Goldstein. 1980. Generation of a chemotactic lipid from arachidonic acid by exposure to a superoxide-generating system. Inflammation. 4:313-328.

35. Petrone, W. F., D. K. English, K. Wong, and J. M. McCord. 1980. Free radicals and inflammation: superoxide-dependent activation of neutrophil chemotactic factor in plasma. Proc. Natl. Acad. Sci. USA. 77:1159-1163. 